\title{
The Influences of Thermoplastic Polymers on the Electrical Efficiency if Used as Material Insulation in Cables
}

\author{
Satar Habib Mnaathr ${ }^{1, *}$, Hayder Saher Naema AL-Hseenawy ${ }^{2}$ \\ ${ }^{1}$ Ministry of Industry and Minerals, Iraq \\ ${ }^{2}$ UR State Company, Iraq
}

Copyright (C) 2014 Horizon Research Publishing All rights reserved.

\begin{abstract}
The chemical synthesis industry jumps high steps with the technological development in this filed, polymeric, Insulation Material (IM), particularly extruded material, progressively switch traditional PILC cable (paper-Isolation lead covered) to become a new popular kind of cable. Many potential candidates, but a popular material is XLPE, due to of its excellent electrical characteristics also dielectric characteristics. Anyway, the material doesn't suitable to ease of recycling. In recent, there is attention shown in utilize material that is easily recycled. Thermoplastic material looks an automatic contender, so the needing for understanding the electrical effectiveness and performance of these materials so important. This paper will show the benefits of thermosetting material XLPE used as cable isolation and the types of thermoplastics with variety of stresses. Voltage stress, environmental stress, and thermal stress are most common stresses considered for material efficiency. As well as, relationships between various stresses and select the benefits of thermosetting materials (XLPE) as cable insulation.
\end{abstract}

Keywords Insulation Materials (IM), Material Efficiency, Thermoplastic Material, Stresses, Electrical Characteristics, XLPE

\section{Introduction}

Date of usage Underground cable back to the time when telegraph was used, during this period several commercial devices to transmit signals became fashionable which led to the widespread underground power system in process worldwide today [1].Underground cables have essential role in power system. With the ongoing advancement of cable qualities, the efficiency of cable IM has supports many enhancements. The voltage ranges of three-phase cables in power systems can be separate into many categories. The demands for cable isolation are different with voltage levels. so there are four levels of voltage in power system, that are extra high voltage $\left(U_{n}>150 \mathrm{kV}\right)$ high voltage $(45 \mathrm{kV} \leq U n \leq$ $150 \mathrm{kV})$, medium voltage $(1 \mathrm{kV} \leq U n \leq 45 \mathrm{kV})$, low voltage $(U n \leq 45 \mathrm{kV}$ ),[2].higher level of the voltage is, the more complex the cable structure and the higher the cable isolation requirement.

At first, PILC cable (paper-insulated lead covered) is the main kind of underground cable used in the countries of world before to the year 1968 for medium and high voltage levels. Paper-insulated lead covered cable is reliable and has a long service history. On the other hand, PILC cable is heavy and it is changed gradually by polymeric cables, among which, the main important role for extruded material. Compared with paper-insulated lead covered cable, extruded cable manufacturing operation will decrease defects in cable structure and that enhances the aging process. Accordingly, extruded IM became the new favorite kind of cable utilized by industry because of its low cost and light weight. The extruded materials most commonly are polyethylene and cross linking polyethylene. With the growing in technological regarding of the chemical synthesis industry, low-voltage power cables are mainly used with polymeric insulation based on composites of rubber Wherever possible he technology is more sophisticated for using in high-voltage electrical cables [3].

In spite of the deterioration of the polymer is to some extent are well understood, there is still research interests in the areas of rapid growth of the trees in the polymer under different stress conditions[3]. Tree of water grow when there is tension voltage and humidity in the system at the same time and will be accelerated when there are pollutants as well $[2]$.

The role of IM is to provide sufficient protection for each of the conductors as well as the closest layer shielding. Any company try to provide conductor with high quality, have treated to protect the conductor from, water, acid, and other factors of environment. To furnish for the closest shielding layer, it is necessary to protect that from the stress of voltage by the conductor. 
Most popular IM utilized in the cable under the ground in all parts of the world is cross-linked polyethylene (XLPE) with electrical characteristics and excellent physical [4]. Despite XLPE insulated cable underground occupies big Percentage in all parts of the world, it is a heat material that cannot be easily recycled, causing the issues of environment, and therefore, it is important to examine and fix some of the recyclable materials to replace XLPE. Thermoplastic material (TM) is a best example about recycling material.

Before selecting a candidate material, it is essential to focus and understand the role of insulation in the structure of the cable; the characteristics of various materials and the potential stress faced by insulators to assist create the criteria for the selection of IM.

\section{The Extruded Materials in Cable Insulation}

Layer of the insulation is mostly located between the connector and the closest layer shielding or the closest electrical ground, Figure 1.

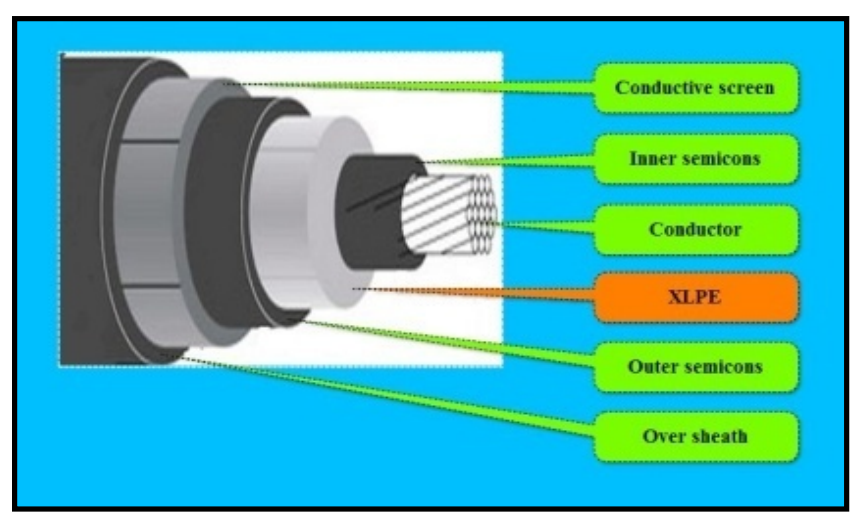

Figure 1. Cable structure

There are two types of Polymeric materials, thermoplastic material and Thermoplastic material

\subsection{Thermoplastic Materials}

Several categories from Thermoplastic materials [5]:

1. Non-polar thermoplastics: Plastic non-polar covalent, is usually symmetrical molecules, like this materials do not exist dipoles polar current as well as application an electric field still not try to align the dipoles. Electrons move slightly towards the electric field to generate the electron polarization, in this situation is that the only traffic of electrons and this is effectively momentary. PTFE is an Examples of non-polar plastic (and several other fluoropolymers as well), PS, PE, and PP, it materials have a propensity to have a low dielectric constant and high impedance. Polymer structure identifies whether non-polar or polar, and this identifies a lot of the dielectric properties of the polymer [6]. In non-polar polymers, there are clearly areas of high and low temperatures for breakdown properties [2, 7]. Curve of temperature dependence show in figure 2.

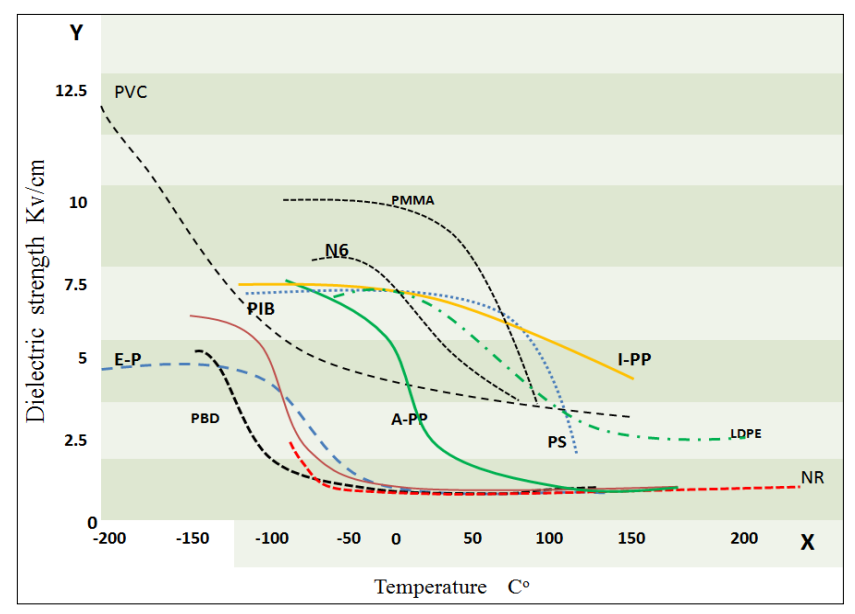

Figure 2. Curve of temperature dependence for non-polar polymers

2. Polar thermoplastics: Polar thermoplastics do not have covalent bonding fully, and there is little disruption in the molecule's electronic charge. In plastic polar, dipoles are generated from an imbalance in the distribution of electrons in the presence of an electric field on the dipoles will be trying to move in line with the electric field [8]. This will generate a "dipole polarization" of the material, and due to the dipoles movement, are involved as well there is a time factor to the movement. Some Examples about plastic polar PVC, PMMA, PC,PA (Nylon), , and such as this materials mostly tend to be only good as insulators. For polar polymers, there is no clearly defined area of low temperature [9]. Curve shows the temperature dependence in figure 3.

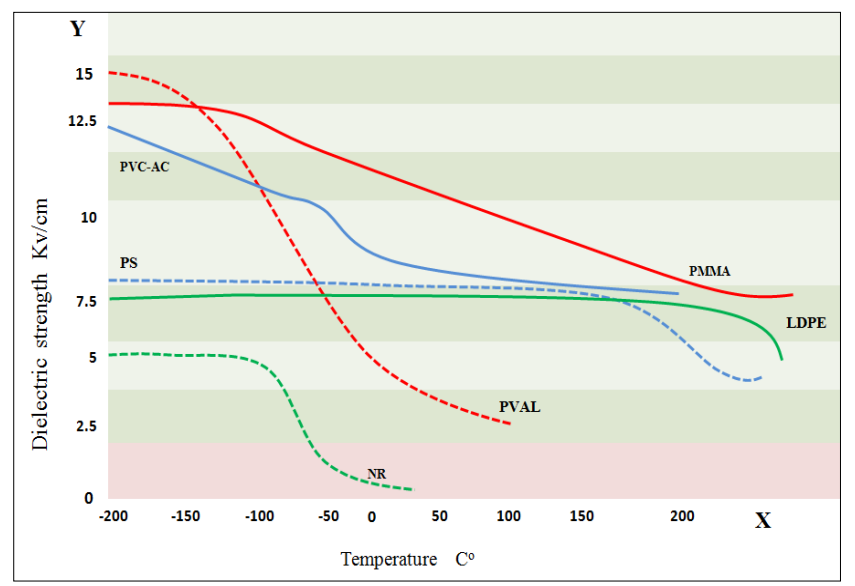

Figure 3. Polar polymers and Temperature dependence

3. Semi-crystalline thermoplastics: have a position between amorphous materials and crystalline materials. thus, the numerical value to each Characteristic is between the range of value of amorphous materials and crystalline materials. Such as polyamide (nylon 6 and nylon 66) and polyester polybutylene terephthalate (PBT) [5]. So the representation of material polar or non-polar utilize in addition to that for the classification of materials when considering the effort stresses. 
4. Amorphous thermoplastic materials: thermoplastic material characteristics of amorphous completely different from the crystalline thermoplastic material. No order of the molecules in the structure of amorphous thermoplastic material, and therefore, it is difficult molding amorphous materials of crystalline materials [5]. Models of amorphous thermoplastic material such as polymethyl methacrylate (PMMA), polycarbonate (PC), polystyrene (PS), acrylonitrile butadiene styrene (ABS), polyphenylene oxide (PPO).

5. Crystalline thermoplastics: In structure of a crystalline thermoplastic the molecules are arranged on a regular basis, Fig.4. With temperature increasing, molecules will get higher energy to break the existing ranking and the crystalline structure will be converted into an amorphous structure. but ,step by step with cooling down, the molecular structure hard to return to the first case or the original. The crystalline area percentage is based on the temperature and how fast the temperature changed. If cooled fast, majority of the molecules have is not sufficient time to reply to change and output crystalline area will come small. But if the changing of temperature is slowly, will retain most of crystalline area. Without a doubt, will be retained sample thicker crystalline area, due to of non-uniform temperature difference versus with a thinner sample. Crystalline thermoplastics have high melting temperatures and more clear-cut. such as materials polyethylene (PE) and polypropylene (PP).
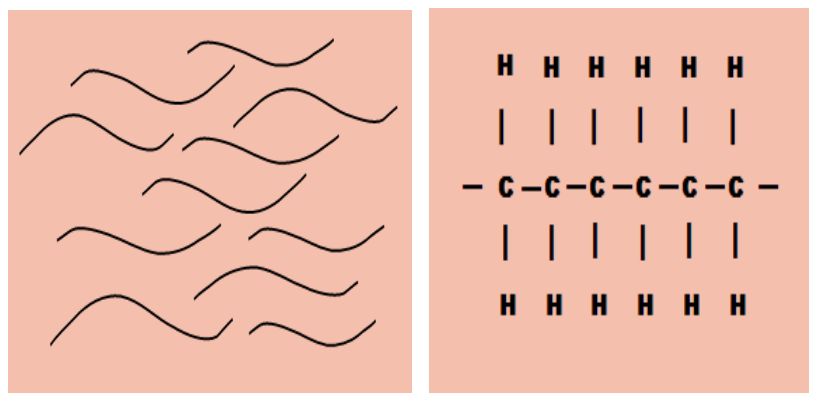

Figure 4. Polyethylene with its Molecular structure.
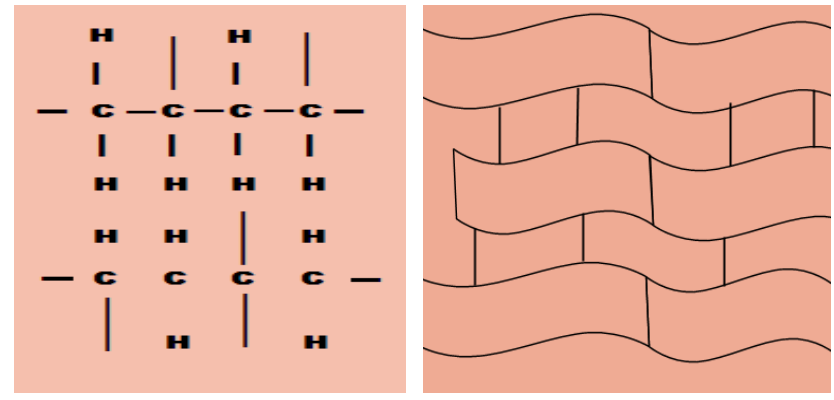

Figure 5. Molecular structure of XLPE

\subsection{Thermosetting Materials}

Thermoplastic materials, Can be a raw material for the manufacture of Thermosetting materials for instance, the thermosetting material via linked a typical insulation material such polyethylene (XLPE), and it is making from polyethylene, as well as it is a thermoplastic material. Under effect the temperature and high pressure condition and organic Peroxide as additives, by linking structure is produced between individual molecular chains. Thus will occur fundamental changes for thermoplastic material to thermosetting material $[4,10]$, as shown in figure 4,5 .

Table 1. Comparison between XLPE with other types of cables [8]

\begin{tabular}{|c|c|c|c|c|c|}
\hline \multicolumn{2}{|c|}{ Characteristics } & XLPE Cable & PVC Cable & EPR Cable & PE Cable \\
\hline \multirow{3}{*}{$\begin{array}{c}\text { Tensile } \\
\text { strength } \\
\left(\mathrm{kg} / \mathrm{mm}^{2}\right)\end{array}$} & Normal & 90 & 70 & 90 & 70 \\
\hline & Overload & 130 & 100 & 130 & 90 \\
\hline & Short circuit & 250 & 160 & 250 & 200 \\
\hline \multicolumn{2}{|c|}{ Tensile strength $\left(\mathrm{kg} / \mathrm{mmm}^{2}\right)$} & 1.9 & $1.0-2.5$ & 0.95 & 1.4 \\
\hline \multicolumn{2}{|c|}{ Elongation (\%) } & $200-350$ & $200-4500$ & $250-550$ & $600-650$ \\
\hline \multicolumn{2}{|c|}{$\begin{array}{c}\text { Volume resistivity at } 20 \mathrm{deg} \\
\mathrm{C}(\Omega . \mathrm{cm})\end{array}$} & 1016 & 1013 & 1015 & 1016 \\
\hline \multicolumn{2}{|c|}{ Dielectric constant, $1 \mathrm{KHz}$} & 2.3 & $4.5-9$ & 3 & 2.3 \\
\hline \multicolumn{2}{|c|}{$\begin{array}{l}\text { Dielectric strength } \\
(\mathbf{K v} / \mathbf{m m})\end{array}$} & 20 & $15-20$ & $15-20$ & 20 \\
\hline
\end{tabular}

With the structure of cross-linking, XLPE has significantly improved buffer property on the existing super electrical features of polyethylene. XLPE insulation material is high and so good. Regarding data from table 1, the XLPE advantages will be extracted as follows:-

1. XLPE has higher resistance to pollutants. This feature is very important for cables which use underground. In water, acid, soil, etc..., Will influence the cable performance when they penetrate into and influence the insulation material performance.

2. XLPE has higher solvent resistance such as oil, weather, inorganic chemicals, organic and chemicals the benefits are shown clearly in Table 1.

3. XLPE has higher temperature stamina, as well as, it up to $\mathrm{C} \mathrm{q} 90$ can operate, that is, even under error condition when higher current flows through the cable, moreover breakdown of cable will have the less risk.

4. XLPE has higher aging resistance within broad of temperatures, that is, it will have longer aging and service compared with other kinds of material.

5. XLPE has Excellent electrical features, It has a higher size resistance, less dielectric constant under $1 \mathrm{kHz}$, higher dielectric strength, which means, less current flowing via cable insulation, lower energy dissipation and within the same voltage stress, lower temperature will be faced by the cable insulation. Thus, the material is utilized in the fields of high voltage applications.

Thus, the disadvantage and drawbacks of thermoplastic material comes in the foreground under the current environmental circumstances by linking that is led to make the thermoplastic material is not be easily recycled albeit with high temperature. so the increased emphasis on recycling and eco-friendly materials, it would not be the 
preferred thermoplastic material so it is advisable to examine and investigate a substitution for XLPE $[2,5,11]$. So thermoplastic material is a good candidate from the perspective of recycling,

\section{Insulation Materials Versus Important Stresses}

Before selecting insulation materials, it is essential to consider stresses that facing the materials. Three types of stresses, environmental stresses, electrical stresses, and thermal stresses. First, environmental stresses composed of the soil contaminants, like acid, water, etc..., which may cause damaging and which might result to breakdown of the cable in the end. With voltage stresses in the high moisture, it led to create water trees, and finally deterioration the dielectric properties of polymer.

Second, electric stress is very important for cable insulation. Thus, the main role of cable insulation represents in voltage endurance, cable insulator is located between cable jacket and conductors which should show excellent electrical characteristics, like, low dissipation factor, high volume resistance, and high breakdown strength. Third, thermal stresses the exponential law and Inverse-power law are the common manifestations in terms of electrical deterioration, while the Arrhenius form is the normal approach applied for thermal deterioration $[12,13]$.

\section{Conclusions}

Based on perspective of assumption in terms of replacing XLPE, this study concluded the benefits of a thermosetting material (XLPE) as cable insulation to afford a benchmark. Intrinsic characteristics of thermoplastics were also concluded to help recognize possible replacements for XLPE. It will then be investigated for their appropriateness by subjecting them to various stresses including environmental stress, voltage stress and thermal stress, moreover the relationship among stresses and equations were established as well.

\section{Acknowledgements}

With highest grateful to Minister of Industry and Minerals in Iraq and all employees with main manager of UR state company for their helping and facilitating everything to accomplished this study and their appreciation. As well as I'm very grateful to some lecturers from Physics Dept at Thi-qar university in Iraq for their constructive suggestions to improve this study.

\section{REFERENCES}

[1] R. de Lieto Vollaro, L. Fontana, and A. Vallati, "Experimental study of thermal field deriving from an underground electrical power cable buried in non-homogeneous soils," Applied Thermal Engineering, vol. 62, pp. 390-397, 2014.

[2] W. Zhao, W. H. Siew, and M. J. Given, "The electrical performance of thermoplastic polymers when used as insulation in cables," in Power Engineering Conference (UPEC), 2013 48th International Universities', 2013, pp. 1-4.

[3] S. Chakravorti, D. Dey, and B. Chatterjee, "Partial Discharge Measurement and Analysis," in Recent Trends in the Condition Monitoring of Transformers, ed: Springer, 2013, pp. 61-115.

[4] Y. Xu, P. Luo, M. Xu, and T. Sun, "Investigation on insulation material morphological structure of 110 and 220 kV XLPE retired cables for reusing," Dielectrics and Electrical Insulation, IEEE Transactions on, vol. 21, pp. 1687-1696, 2014.

[5] E. A. Cherney, "50 years in the development of polymer suspension-type insulators," Electrical Insulation Magazine, IEEE, vol. 29, pp. 18-26, 2013.

[6] M. Arjmand, "Electrical Conductivity, Electromagnetic Interference Shielding and Dielectric Properties of Multi-walled Carbon Nanotube/Polymer Composites," 2014.

[7] I. M. Ward and J. Sweeney, Mechanical properties of solid polymers: John Wiley \& Sons, 2012.

[8] M. J. Kendall and C. R. Siviour, "Rate dependence of poly (vinyl chloride), the effects of plasticizer and timetemperature superposition," Proceedings of the Royal Society A: Mathematical, Physical and Engineering Science, vol. 470, p. 20140012, 2014.

[9] E. Logakis, L. Petersson, and J. Viertel, "Dielectric spectroscopy and thermally stimulated depolarization current investigations in low density polyethylene," in Solid Dielectrics (ICSD), 2013 IEEE International Conference on, 2013, pp. 948-951.

[10] K. Barber and G. Alexander, "Insulation of electrical cables over the past 50 years," Electrical Insulation Magazine, IEEE, vol. 29, pp. 27-32, 2013.

[11] S. Amin and M. Amin, "Thermoplastic elastomeric (TPE) materials and their use in outdoor electrical insulation," Rev. Adv. Mater. Sci, vol. 29, pp. 15-30, 2011.

[12] L. Simoni, "A general approach to the endurance of electrical insulation under temperature and voltage," Electrical Insulation, IEEE Transactions on, pp. 277-289, 1981.

[13] D. Lenko, S. Schlögl, S. Bichler, G. Lemesch, F. Ramsauer, W. Ladstätter, J. Rosc, and W. Kern, "New approaches towards the investigation on defects and failure mechanisms of insulating composites used in high voltage applications," Composites Part B: Engineering, vol. 58, pp. 83-90, 2014. 